July 8,2003

\title{
Spin 1/2 Particle on a Cylinder with Radial Magnetic Field
}

\author{
Chryssomalis Chryssomalakos, \\ Instituto de Ciencias Nucleares \\ Universidad Nacional Autónoma de México \\ Apdo. Postal 70-543, 04510 México, D.F., MEXICO \\ chryss@nuclecu. unam.mx
}

\author{
Alfredo Franco, and Alejandro Reyes-Coronado \\ Instituto de Física \\ Universidad Nacional Autónoma de México \\ Apdo. Postal 20-364, 01000 México, D.F., MEXICO \\ alfredof@fisica.unam.mx, coronado@fisica.unam.mx
}

\begin{abstract}
We study the motion of a quantum charged particle, constrained on the surface of a cylinder, in the presence of a radial magnetic field. When the spin of the particle is neglected, the system essentially reduces to an infinite family of simple harmonic oscillators, equally spaced along the axis of the cylinder. Interestingly enough, it can be used as a quantum Fourier transformer, with convenient visual output. When the spin $1 / 2$ of the particle is taken into account, a non-conventional perturbative analysis results in a recursive closed form for the corrections to the energy and the wavefunction, for all eigenstates, to all orders in the magnetic moment of the particle. A simple two-state system is also presented, the time evolution of which involves an approximate precession of the spin perpendicularly to the magnetic field. A number of plots highlight the findings while several three-dimensional animations have been made available on the web.
\end{abstract}




\section{Contents}

\begin{tabular}{lll}
\hline & Introduction & 2
\end{tabular}

\begin{tabular}{|lll}
\hline 2 & The Spinless Case & 3
\end{tabular}

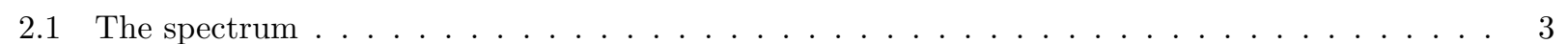

2.2 A quantum Fourier transformen $\ldots \ldots \ldots \ldots \ldots \ldots \ldots$

3 The Spin-1/2 Case $\quad 4$

3.1 Separation of variables $\ldots \ldots \ldots \ldots \ldots \ldots \ldots \ldots$

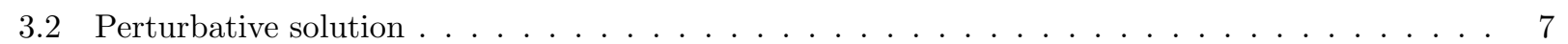

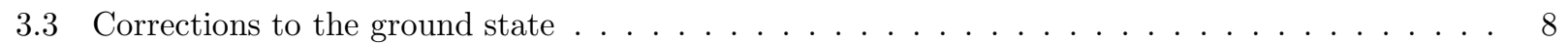

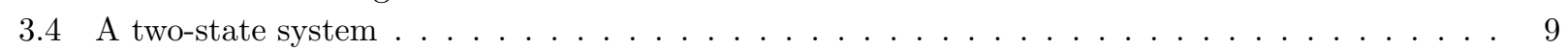

\begin{tabular}{|lrl}
4 Concluding Remarks & 9
\end{tabular}

A First Order Corrections to the Ground State: the Standard Treatment 10

\section{Introduction}

The quantum mechanical description of the motion of charged particles in a magnetic field is a classic application of the theory, having given rise to some of its most striking results. Among them, the seminal analysis by Dirac [5], of the motion in the field of a magnetic monopole, continues to inspire decades after its inception, and motivates the study of similar quantum systems that share the characteristic of providing insights into the fundamentals without too much distraction by analytical complexity. Such systems are invaluable pedagogically, as they furnish a manageable, yet captivating testing ground of the fundamentals of the theory.

The problem of the motion of a non-relativistic quantum particle in a plane, in the presence of a perpendicular homogeneous magnetic field is presented in several textbooks (see, e.g., 13]) - nevertheless, it seems to be the only standard example of this type available. The main purpose of this paper is to draw attention to the fact that the analogous problem for the cylinder is also manageable, even when augmented to include a spin $1 / 2$. In this latter case, we also show how the use of the creation and anihilation operator machinery greatly simplifies the perturbative analysis of the problem, in comparison to the standard textbook procedure.

Despite the simplicity of the problem and it being an obvious variation on the monopole theme, we have not been able to find a treatment in the literature. The motion of a spin- $1 / 2$ particle in the field of a magnetic monopole has been studied in detail, both in the non-relativistic [1, 17, 8, 15, 9] and relativistic [12] cases. Symmetry aspects of the problem have also been considered extensively (see, e.g., [11]), with the discovery of an underlying supersymmetry among the most notable results [4, 2, 10. On the other hand, quantum spinless particles moving on curves or surfaces have been extensively studied (see, e.g., 3] 16, 14] and references therein) with a general discussion of the effects of a vector potential given in [6]. It is our hope that the use of the above simple system will enhance the exposition of this fascinating part of the theory. It should also be of interest in practical applications, such as constrained quantum mechanics and carbon nanotube physics.

Consider a classical charged particle, constrained to move on the surface of an infinite cylinder, in the presence of a radial magnetic field,

$$
\vec{B}(\vec{r})=B_{0} \frac{a}{\rho} \hat{\rho}
$$


where $a$ is the radius of the cylinder and $B_{0}$ is the field strength on its surface ${ }^{1}$. The equations of motion for the particle are

$$
m \dot{v}_{z}=-q B_{0} v_{\phi}, \quad m \dot{v}_{\phi}=q B_{0} v_{z}
$$

where $m, q$ are the mass and charge of the particle respectively and $\{\hat{\rho}, \hat{\phi}, \hat{z}\}$ is a right-handed basis. The solutions to (2) are two simultaneous oscillations: the momentum $p_{z}$ of the particle oscillates like, say, $\cos (\omega t)$ (with $\omega=q B_{0} / m c$ ) while its angular momentum along the $z$-axis oscillates like $\sin (\omega t$ ). Thus, the particle's kinetic energy oscillates between a linear and a rotational form, becoming, for example, purely rotational at the turning points of the oscillation along $z$.

We study, in this paper, the quantum mechanical version of the above problem, adding, at a later stage, a spin- $1 / 2$ to the particle. The treatment of the spinless case, contained in Sect. 22 is exact - the problem separates and reduces to an infinite collection of harmonic oscillators along $z$. We find, nevertheless, the resulting quantum system particularly rich and with surprising properties - it functions, for example, as a quantum Fourier transformer with convenient visual output (see Sec. 2.2). The addition of spin is treated perturbatively in Sect. 3 with a non-conventional method that greatly simplifies the calculations. We are able to give recursion relations for the corrections to the wavefunctions and the energy to all orders, for all unperturbed eigenstates, and apply the results to compute second-order corrections to the ground state. Several plots highlight the findings. We also make available on the web several three-dimensional color animations of the time evolution of the wavefunction, with or without spin, and corresponding to various initial conditions. An appendix shows how the standard perturbation theory treatment of the problem reproduces, albeit laboriously, our first order results.

\section{The Spinless Case}

\subsection{The spectrum}

The magnetic field of Eq. (10) can be obtained, in the vicinity of the surface of the cylinder, from the vector potential

$$
\vec{A}(\vec{r})=-B_{0} \frac{a}{\rho} z \hat{\phi}
$$

The Hamiltonian for a quantum spinless particle constrained to move on the surface of the cylinder is given by

$$
\begin{aligned}
\hat{H} & =\frac{1}{2 m}\left(\vec{p}-\frac{q}{c} \vec{A}\right)^{2} \\
& =-\frac{\hbar^{2}}{2 m}\left(\frac{1}{a^{2}} \partial_{\phi}^{2}+\partial_{z}^{2}\right)+\frac{q^{2} B_{0}^{2}}{2 m c^{2}} z^{2}-i \frac{\hbar q B_{0}}{m c a} z \partial_{\phi} .
\end{aligned}
$$

The wavefunction $\Psi(\phi, z)=\frac{1}{\sqrt{2 \pi}} e^{i \ell \phi} Z(z)$ is an eigenfunction of $\hat{H}$, with eigenvalue $E$, provided $Z(z)$ satisfies (primes denote differentiation w.r.t. $z$ )

$$
-\frac{\hbar^{2}}{2 m} Z^{\prime \prime}(z)+\frac{1}{2} m \omega^{2}(z+\ell b)^{2} Z(z)=E Z(z),
$$

where we have set

$$
\omega=\frac{q B_{0}}{m c}, \quad b=\frac{\hbar c}{q B_{0} a},
$$

and, in what follows, we take $\hbar=m=\omega=1$. This is the equation for a simple harmonic oscillator (SHO), centered at $z=-\ell b$. We conclude that, for each integer value of $\ell$, one obtains a copy of the usual SHO

\footnotetext{
${ }^{1}$ Such a radial field can be thought to be produced by a homogeneous linear magnetic charge density, or, more realistically, in the exterior of a solenoid of radius $R, R<a$, placed along the axis of the cylinder and carrying surface current density $\vec{J}=-\frac{2 B_{0} a}{\mu_{0} R^{2}} z \hat{\phi}$
} 
spectrum, centered at $z=-\ell b$, i.e., the eigenfunctions and eigenvalues of $\hat{H}$ are given by

$$
\langle\phi, z \mid n, \ell\rangle=N_{n} H_{n}(z+\ell b) e^{-(z+\ell b)^{2} / 2} \frac{1}{\sqrt{2 \pi}} e^{i \ell \phi}, \quad E_{\ell, n} \equiv E_{n}=n+\frac{1}{2}, \quad N_{n} \equiv\left(2^{n} n ! \sqrt{\pi}\right)^{-\frac{1}{2}},
$$

where $|n, \ell\rangle$ denote the corresponding eigenkets $(n=0,1,2, \ldots ; \ell \in \mathbb{Z})$ and $H_{n}(z)$ are the Hermite polynomials.

\subsection{A quantum Fourier transformer}

Suppose that the wavefunction of the particle, at $t=0$, is given by ${ }^{2}$

$$
\Psi_{\ell}(\phi, z, t=0) \equiv\langle\phi, z \mid 0,0, \ell\rangle=N_{0} e^{-z^{2} / 2} \frac{1}{\sqrt{2 \pi}} e^{i \ell \phi} .
$$

If $\ell=0$, we have one of the infinitely many ground states of the system and the time evolution is by a phase factor. Consider now the case $\ell \neq 0$. Then the $z$-part "sees" a quadratic potential centered at $z=-\ell b$ but the initial wavefunction is a gaussian centered at the origin. This is a coherent state and its time evolution is an oscillation around $z=-\ell b$, with the frequency $\omega=1$ of the oscillator,

$$
\Psi_{\ell}(\phi, z, t)=N_{0} e^{-i t / 2} e^{-i(z+\ell b) \ell b \sin t} e^{-(z+\ell b(1-\cos t))^{2} / 2} \frac{1}{\sqrt{2 \pi}} e^{i \ell \phi} .
$$

Notice that a physically irrelevant global phase factor $e^{i \ell^{2} b^{2} / 4 \sin 2 t}$ has been omitted from the above expression. We may now exploit linearity to write down the time evolution of a gaussian (in $z$ ), centered at the origin, with arbitrary $\phi$-dependence,

$$
\Psi(\phi, z, t=0)=N_{0} e^{-z^{2} / 2} f(\phi)
$$

Writing

$$
f(\phi)=\sum_{\ell=-\infty}^{\infty} f_{\ell} e^{i \ell \phi}
$$

we obtain,

$$
\Psi(\phi, z, t)=\sum_{\ell=-\infty}^{\infty} f_{\ell} \Psi_{\ell}(\phi, z, t)
$$

i.e., each Fourier mode of $f(\phi)$ gives rise to a gaussian in $z$, oscillating like a coherent state around $z=-\ell b$ with frequency $\omega=1$. Taking $b \gg 1$, so that the various gaussians separate after half a period, converts the system to a quantum Fourier transformer with convenient visual output: looking at the wavefunction at time $t=\pi$ (a half-period), one sees the above gaussians at the (second) turning point of their oscillation, at $z=-2 \ell b$, with their amplitudes proportional to the Fourier amplitudes $f_{\ell}$. In Fig. [1 we plot several frames of the time evolution of $\Psi$, when $f(\phi)=\cos \phi$ - the last frame, at $t=\pi$, clearly displays the Fourier content of $f$. Fig. 2 corresponds to the initial wavefunction $\Psi(\phi, z, t=0) \sim e^{-z^{2} / 2}\left(1+e^{-i \phi}+1.5 e^{-i 2 \phi}+e^{-i 3 \phi}\right)$.

\section{The Spin-1/2 Case}

\subsection{Separation of variables}

For a spin-1/2 particle, the wavefunction has two components, $\Psi_{+}(\phi, z), \Psi_{-}(\phi, z)$, which we arrange in a column vector. The spin interacts with the magnetic field via $\hat{H}_{\mathrm{int}}=-\lambda \vec{S} \cdot \vec{B}$, which, for the field given in (1)

\footnotetext{
${ }^{2}$ We use the notation $\left.\langle\phi, z \mid n, \ell, m\rangle=N_{n} H_{n}(z) \ell b\right) e^{-(z+\ell b)^{2} / 2} \frac{1}{\sqrt{2 \pi}} e^{i m \phi}$ (not to be confused, hopefully, with the standard spherical symmetry notation) - these wavefunctions are eigenfunctions of $\hat{H}$ only when $m=\ell$, in which case they will be denoted by $|n, \ell\rangle$, as above.
} 

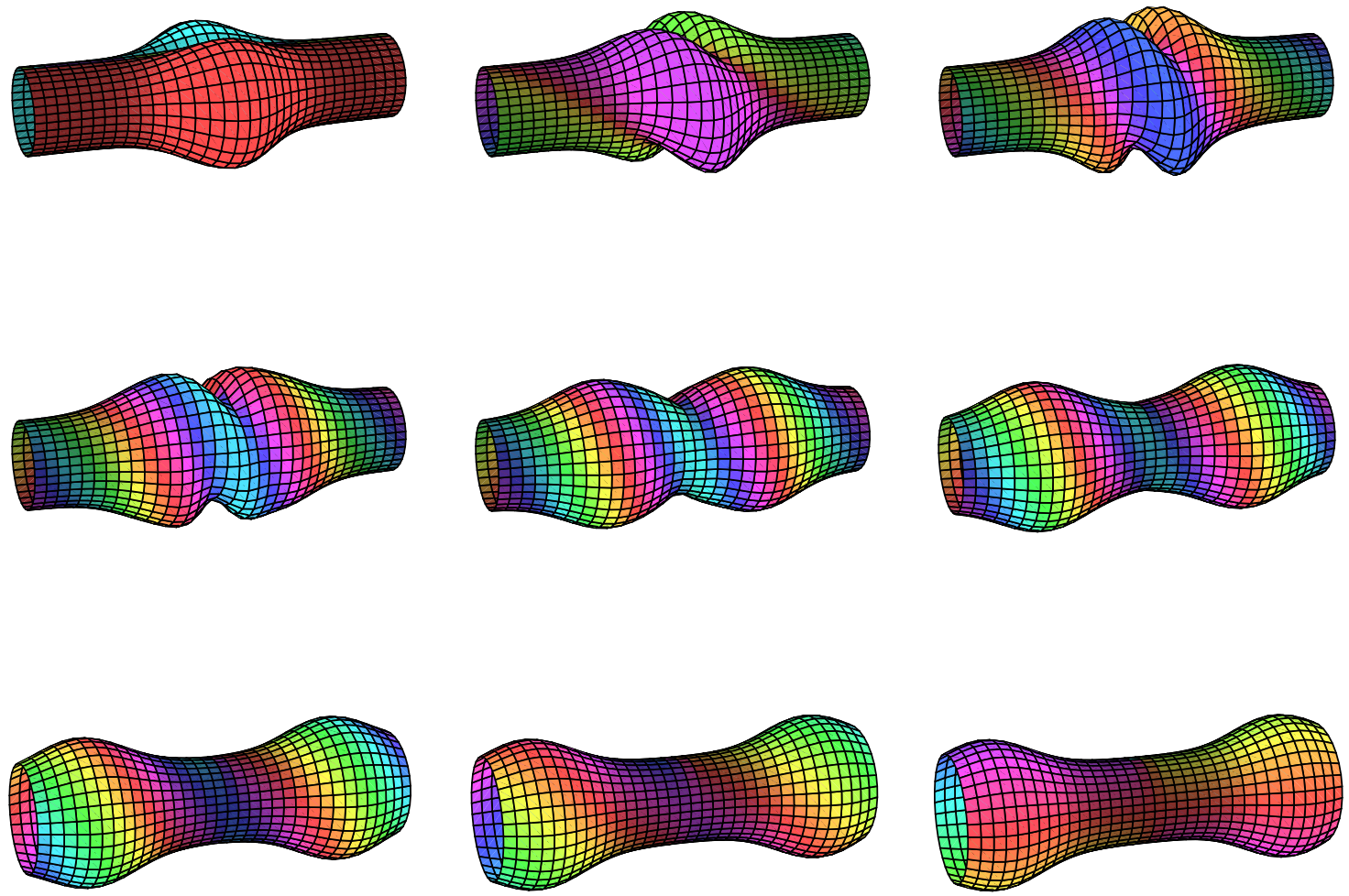

Figure 1: Time evolution of the initial wavefunction $\Psi(\phi, z, t=0)=N_{0} e^{-z^{2} / 2} \cos \phi$. The modulus of $\Psi$ is indicated by the radial distance of the surface from that of the cylinder while its phase is color-coded, with $1, i,-1,-i$ corresponding to red, green, blue, purple (several animations in color, including the above, can be seen at http://www.nuclecu.unam.mx/ chryss). The time $t$ is equal to zero at the top left and increases to the right and downwards, reaching $t=\pi$ (half a period) at the bottom right.

becomes

$$
\hat{H}_{\mathrm{int}}=-\frac{1}{2} \lambda B_{0}\left(\begin{array}{cc}
0 & e^{-i \phi} \\
e^{i \phi} & 0
\end{array}\right) .
$$

In order to achieve separation of variables now, we need to take

$$
\Psi_{+}(\phi, z)=\frac{1}{\sqrt{2 \pi}} e^{i \ell \phi} Z_{+}(z), \quad \Psi_{-}(\phi, z)=\frac{1}{\sqrt{2 \pi}} e^{i(\ell+1) \phi} Z_{-}(z)
$$

The resulting equation for the $Z$ 's is

$$
\begin{aligned}
\hat{H}_{\ell} Z_{+}(z)+\epsilon Z_{-}(z) & =E Z_{+}(z) \\
\hat{H}_{\ell+1} Z_{-}(z)+\epsilon Z_{+}(z) & =E Z_{-}(z),
\end{aligned}
$$

where $\hat{H}_{\ell}$ is a SHO hamiltonian centered at $z=-\ell b$,

$$
\hat{H}_{\ell}=-\frac{1}{2} \partial_{z}^{2}+\frac{1}{2}(z+\ell b)^{2},
$$



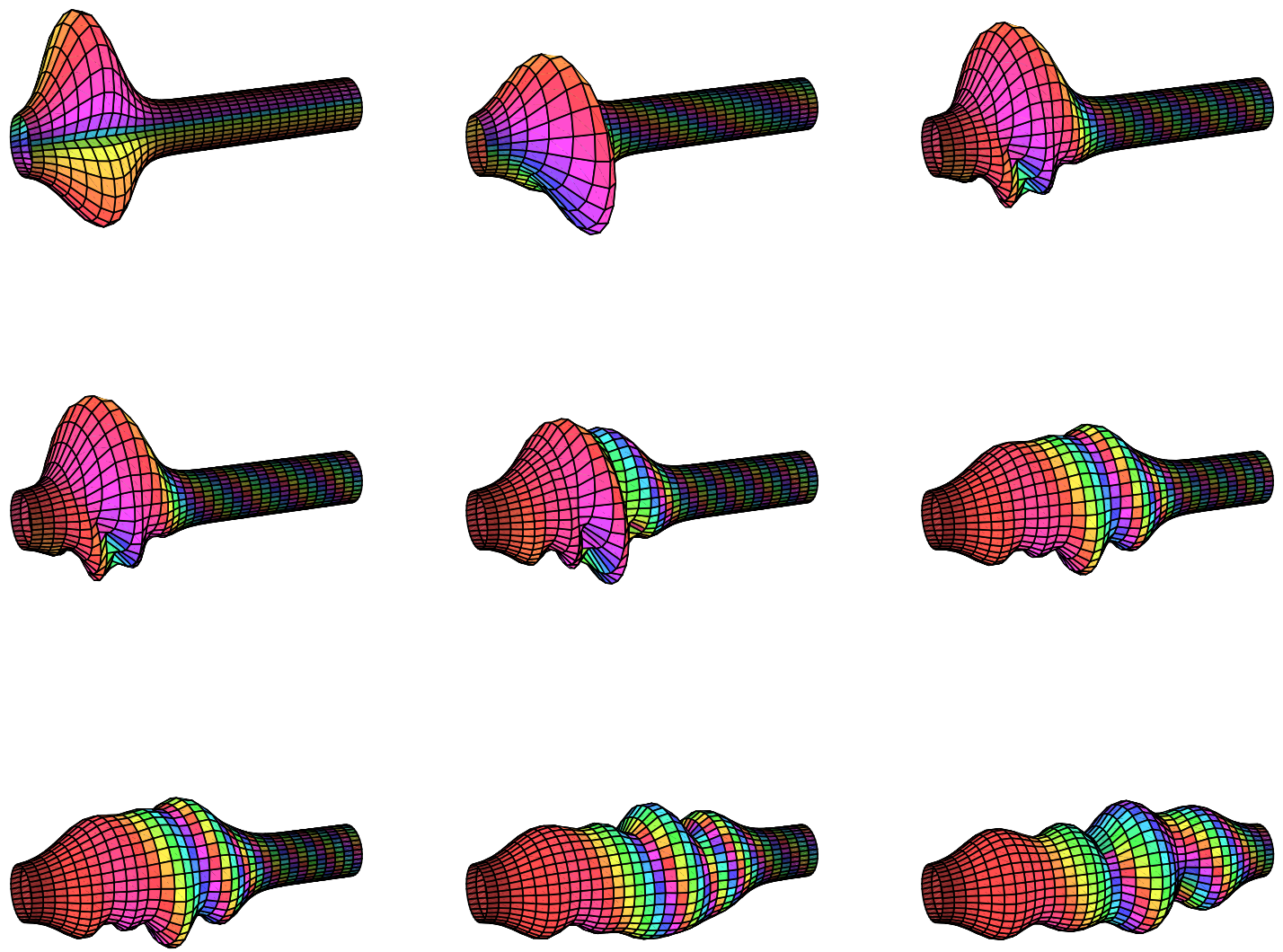

Figure 2: Time evolution of the initial wavefunction $\Psi(\phi, z, t=0) \sim e^{-z^{2} / 2}\left(1+e^{-i \phi}+1.5 e^{-i 2 \phi}+\right.$ $\left.e^{-i 3 \phi}\right)$. Conventions are as in Fig. 1] Notice how the amplitudes of the gaussians in the last frame correspond to the Fourier components of the initial wavefunction.

and $\epsilon \equiv-\hbar \lambda B_{0} / 2$. We see that the problem reduces to that of two SHO's, a distance $b$ apart, coupled by the $\epsilon$ terms in (15). Our task is to solve (15) perturbatively in $\epsilon$. Once the solutions are known, to a certain order in $\epsilon$, we can form the spinor

$$
\Psi(\phi, z)=\left(\begin{array}{c}
\Psi_{+}(\phi, z) \\
\Psi_{-}(\phi, z)
\end{array}\right)=\left(\begin{array}{c}
\frac{1}{\sqrt{2 \pi}} e^{i \ell \phi} Z_{+}(z) \\
\frac{1}{\sqrt{2 \pi}} e^{i(\ell+1) \phi} Z_{+}(z)
\end{array}\right)
$$

from which the probability density and spin direction can be extracted as

$$
\rho=\sqrt{\left|Z_{+}\right|^{2}+\left|Z_{-}\right|^{2}}, \quad \alpha=2 \arctan \frac{\left|Z_{-}\right|}{\left|Z_{+}\right|}, \quad \beta=\operatorname{Im}\left(\log \frac{\Psi_{-}}{\Psi_{+}}\right),
$$

where the spin direction $\hat{n}$ is given by $\hat{n}=(\sin \alpha \cos \beta, \sin \alpha \sin \beta, \cos \alpha)$, in Cartesian coordinates. The solutions of (15) have no relative (complex) phase and can be taken real. Then, on the $\phi=0$ plane, the spin lies in the $x-z$ plane. The extra $e^{i \phi}$ factor in $\Psi_{-}$guarantees that when we change our position on the 
cylinder by $\phi$, the spin also rotates by the same angle and its direction is therefore obtained by revolution of the $\phi=0$ configuration, in other words, $\beta=\phi$ in (18). These remarks are of course valid only for the energy eigenstates - the time evolution of general states results in the spin pointing outside of the radial plane as well, even if they start within it.

\subsection{Perturbative solution}

One may treat the system of the two coupled differential equations in (15) by standard perturbation theory methods - we give the first-order analysis along these lines in the appendix. It is instructive though, as well as much more efficient, to exploit the SHO machinery of raising and lowering operators. We begin by transforming (15) into a single differential-difference equation. Indeed, it is clear from the symmetry of these equations that the solutions can be taken to satisfy $Z_{-}(z)= \pm Z_{+}(-z-b)$ - we will refer to the two possibilities as symmetric and antisymmetric respectively. Taking $\ell=0$ and restricting to the symmetric case, the first of (15) becomes

$$
\hat{H}_{0} Z_{\mathrm{s}+}(z)+\epsilon Z_{\mathrm{s}+}(-z-b)=E Z_{\mathrm{s}+}(z) .
$$

We now write $\hat{H}_{0}=a^{\dagger} a+\frac{1}{2}$ and introduce the ket whose wavefunction is $Z_{\mathrm{s}+}(z)$,

$$
Z_{\mathrm{s}+}(z)=\left\langle z \mid Z_{\mathrm{s}+}\right\rangle, \quad\left|Z_{\mathrm{s}+}\right\rangle=\sum_{n=0}^{\infty} c_{n}\left(a^{\dagger}\right)^{n}|0\rangle_{0} \equiv f\left(a^{\dagger}\right)|0\rangle_{0},
$$

where $|0\rangle_{0}$ is the ground state for $\ell$ equal to zero (i.e., centered at the origin) and $f\left(a^{\dagger}\right)$ is defined by the last equation - the idea is that any ket can be obtained by some function of $a^{\dagger}$ applied to the ground state. The wavefunction $Z_{\mathrm{s}+}(-z-b)$ is obtained from $Z_{\mathrm{s}+}(z)$ by first reflecting around the origin and then effecting the translation $z \mapsto z+b$. Reflecting around the origin an eigenfunction of $\hat{H}_{0}$ introduces a sign given by the parity of the state, which shows that the reflected state is produced by $f\left(-a^{\dagger}\right)$ applied to the ground state. The reflected and translated state then is given by

$$
\begin{aligned}
T_{-b} f\left(-a^{\dagger}\right)|0\rangle_{0} & =e^{-b^{2} / 4} e^{-\frac{b}{\sqrt{2}} a^{\dagger}} e^{\frac{b}{\sqrt{2}} a} f\left(-a^{\dagger}\right)|0\rangle_{0} \\
& =e^{-b^{2} / 4} e^{-\frac{b}{\sqrt{2}} a^{\dagger}} f\left(-a^{\dagger}-\frac{b}{\sqrt{2}}\right)|0\rangle_{0},
\end{aligned}
$$

which brings (19) into the form

$$
\left(a^{\dagger} a+\frac{1}{2}\right) f\left(a^{\dagger}\right)|0\rangle_{0}+\epsilon e^{-b^{2} / 4} e^{-\frac{b}{\sqrt{2}} a^{\dagger}} f\left(-a^{\dagger}-\frac{b}{\sqrt{2}}\right)|0\rangle_{0}=E f\left(a^{\dagger}\right)|0\rangle_{0} .
$$

Noting that the $a-a^{\dagger}$ commutation relations are identical to the $\partial_{x}-x$ ones, we may infer a differential difference equation for the function $f(x)$,

$$
x f^{\prime}(x)+\frac{1}{2} f(x)+\epsilon e^{-b^{2} / 4} e^{-\frac{b}{\sqrt{2}} x} f\left(-x-\frac{b}{\sqrt{2}}\right)=E f(x) .
$$

We now specify to the case where the unperturbed state is the $n$-th excited state of the SHO. The perturbed state will be denoted by $f_{n}\left(a^{\dagger}\right)|0\rangle_{0}$, with energy $E_{n}$, where

$$
f_{n}(x)=\sum_{k=0}^{\infty} f_{n}^{(k)}(x) \epsilon^{k}, \quad E_{n}=\sum_{k=0}^{\infty} E_{n}^{(k)} \epsilon^{k} .
$$

Notice that

$$
f_{n}^{(0)}(x)=\frac{1}{\sqrt{n !}} x^{n}, \quad \quad E_{n}^{(0)}=n+\frac{1}{2} .
$$


Substituting these expansions in (23) we obtain

$$
\begin{aligned}
x \partial_{x} f_{n}^{(k)}(x)-n f_{n}^{(k)}(x)= & -e^{-b^{2} / 4} e^{-\frac{b}{\sqrt{2}} x} f_{n}^{(k-1)}\left(-x-\frac{b}{\sqrt{2}}\right)+\frac{1}{\sqrt{n !}} E_{n}^{(k)} x^{n} \\
& +\sum_{m=1}^{k-1} E_{n}^{(k-m)} f_{n}^{(m)}(x),
\end{aligned}
$$

where we separated the $m=0, k$ terms in the sum on the r.h.s. and used (25). Notice that the r.h.s. above only contains $f_{n}^{(m)}$ with $m<k$, so (26) can be used recursively to determine any $f_{n}^{(k)}(x)$.

The requirement that the perturbed eigenket be normalized implies that the corrections, order by order in $\epsilon$, have to be orthogonal to the unperturbed eigenket $|n\rangle_{0}$. This in turn implies that, for $k>0$, the coefficient of $x^{n}$ in $f_{n}^{(k)}(x)$ must vanish. Then so does the coefficient of $x^{n}$ in $x \partial_{x} f_{n}^{(k)}(x)$. Using this information, we can extract the coefficient of $x^{n}$ on both sides of (26) - the resulting equation fixes recursively the energy corrections $E_{n}^{(k)}$,

$$
E_{n}^{(k)}=\frac{1}{\sqrt{n !}} e^{-b^{2} / 4} \sum_{r=0}^{n}\left(\begin{array}{l}
n \\
r
\end{array}\right)\left(-\frac{b}{\sqrt{2}}\right)^{n-r}\left(\partial_{x}^{r} f_{n}^{(k-1)}\right)\left(-\frac{b}{\sqrt{2}}\right) .
$$

This is an appropriate point to comment on the antisymmetric solutions. The difference in this case is that the $\epsilon$ term in (23) appears with a minus sign. Since this is the only place where $\epsilon$ appears explicitly, we conclude that one gets the antisymmetric solutions from the symmetric ones by the substitution $\epsilon \rightarrow-\epsilon$. As we will see later on, symmetric solutions have their spin parallel, more or less, with the magnetic field while antisymmetric ones have it antiparallel. Since $\epsilon$ is proportional to the magnetic moment of the particle, the above statement about the relation between the two kinds of solutions essentially says that the symmetric solution for a particle coincides with the antisymmetric solution for the same particle but with the opposite magnetic moment.

\subsection{Corrections to the ground state}

For $n=0$, Eqs. (26), (27) simplify considerably,

$$
\begin{aligned}
x \partial_{x} f_{0}^{(k)}(x) & =-e^{-b^{2} / 4} e^{-\frac{b}{\sqrt{2}} x} f_{0}^{(k-1)}\left(-x-\frac{b}{\sqrt{2}}\right)+E_{0}^{(k)}+\sum_{m=1}^{k-1} E_{0}^{(k-m)} f_{0}^{(m)}(x) \\
E_{0}^{(k)} & =e^{-b^{2} / 4} f_{0}^{(k-1)}\left(-\frac{b}{\sqrt{2}}\right) .
\end{aligned}
$$

The unitarity argument given above implies in this case that $f_{0}^{(k)}(0)=0$, for all $k$ greater than zero - this fixes the lower integration limit in the solution of (28) equal to zero. The change of variable $x_{k}^{\prime} \rightarrow x s_{k}$ and the substitution of (29) finally give

$$
f_{0}^{(k)}(x)=e^{-b^{2} / 4} \int_{0}^{1} \frac{d s_{k}}{s_{k}}\left\{f_{0}^{(k-1)}\left(-\frac{b}{\sqrt{2}}\right)-e^{-\frac{b}{\sqrt{2}} x s_{k}} f_{0}^{(k-1)}\left(-x s_{k}-\frac{b}{\sqrt{2}}\right)+\sum_{m=1}^{k-1} f_{0}^{(k-m-1)}\left(-\frac{b}{\sqrt{2}}\right) f_{0}^{(m)}\left(x s_{k}\right)\right\} .
$$

Notice that the (apparent) pole of the integrand at $s_{k}=0$ cancels out.

We look in some detail now at the wavefunctions and resulting spin configurations, including up to quadratic corrections. For the first three $f^{(k)}$, Eqs. (25), (30) give

$$
\begin{aligned}
& f_{0}^{(0)}(x)=1 \\
& f_{0}^{(1)}(x)=e^{-b^{2} / 4} \int_{0}^{1} \frac{d s_{1}}{s_{1}}\left(1-e^{-\frac{b}{\sqrt{2}} x s_{1}}\right) \\
& f_{0}^{(2)}(x)=e^{-b^{2} / 2} \int_{0}^{1} \int_{0}^{1} \frac{d s_{2} d s_{1}}{s_{2} s_{1}}\left\{2-e^{b^{2} s_{1} / 2}-e^{-\frac{b}{\sqrt{2}} x s_{2}}+e^{\frac{b^{2}}{2} s_{1}} e^{-\frac{b}{\sqrt{2}} s_{2}\left(1-s_{1}\right) x}-e^{-\frac{b}{\sqrt{2}} s_{2} s_{1} x}\right\}
\end{aligned}
$$


while for the corresponding energy corrections we get

$$
E_{0}^{(0)}=\frac{1}{2}, \quad E_{0}^{(1)}=e^{-b^{2} / 4}, \quad E_{0}^{(2)}=e^{-b^{2} / 2} \int_{0}^{1} \frac{d s_{1}}{s_{1}}\left(1-e^{\frac{b^{2}}{2} s_{1}}\right) .
$$

Applying the above $f$ 's to the ground state and projecting onto the position eigenket $|z\rangle$ we find the wavefunctions

$$
\begin{aligned}
Z_{+}^{(0)}(z)= & N_{0} e^{-z^{2} / 2} \\
Z_{+}^{(1)}(z)= & N_{0} e^{-b^{2} / 4} \int_{0}^{1} \frac{d s_{1}}{s_{1}}\left(e^{-z^{2} / 2}-e^{b^{2} s_{1}^{2} / 4} e^{-\left(z+b s_{1}\right)^{2} / 2}\right) \\
Z_{+}^{(2)}(z)= & N_{0} e^{-b^{2} / 2} \int_{0}^{1} \int_{0}^{1} \frac{d s_{2} d s_{1}}{s_{2} s_{1}}\left\{\left(2-e^{b^{2} / 2 s_{1}}\right) e^{-z^{2} / 2}-e^{b^{2} s_{2}^{2} / 4} e^{-\left(z+b s_{2}\right)^{2} / 2}\right. \\
& \left.+e^{b^{2} s_{1} / 2+b^{2} s_{2}^{2}\left(1-s_{1}\right)^{2} / 4} e^{-\left(z+b s_{2}\left(1-s_{1}\right)\right)^{2} / 2}-e^{b^{2} s_{2}^{2} s_{1}^{2} / 4} e^{-\left(z+b s_{2} s_{1}\right)^{2} / 2}\right\} .
\end{aligned}
$$

In terms of these, the symmetric solution for the spinor has components (we ommit an overall normalization factor)

$$
Z_{\mathrm{s}+}(z)=Z_{+}^{(0)}(z)+\epsilon Z_{+}^{(1)}(z)+\epsilon^{2} Z_{+}^{(2)}(z), \quad Z_{\mathrm{s}-}(z)=Z_{\mathrm{s}+}(-z-b)
$$

and energy

$$
E_{\mathrm{s} 0}=E_{0}^{(0)}+\epsilon E_{0}^{(1)}+\epsilon^{2} E_{0}^{(2)}
$$

while the antisymmetric solution is given by

$$
Z_{\mathrm{a}+}(z)=-Z_{+}^{(0)}(z)+\epsilon Z_{+}^{(1)}(z)-\epsilon^{2} Z_{+}^{(2)}(z), \quad Z_{\mathrm{a}-}(z)=-Z_{\mathrm{a}+}(-z-b)
$$

with energy

$$
E_{\mathrm{a} 0}=E_{0}^{(0)}-\epsilon E_{0}^{(1)}+\epsilon^{2} E_{0}^{(2)} .
$$

Plots of $Z_{ \pm}$, for both cases, as well as the corresponding spin configurations, are given in Figs. 3 4 Notice that the effect of the perturbation is small, despite a rather large value of $\epsilon$. This can be traced to the fact that the parameter values used give rise to a small overlap of two neighboring gaussians. As a result, the range of validity of our perturbative results is considerable larger that the standard $\epsilon \ll 1$.

\subsection{A two-state system}

Consider a state that, at $t=0$, is the sum of the symmetric and antisymmetric $n=0$ states found above, for, say, $\ell=0$. The spin-down component of this state is of order 1 while the spin-up component is of order $\epsilon$. Roughly speaking, the particle is localized at $z=-b$ and its spin points along the negative $z$-axis. Then the standard two-state system analysis shows that the amplitudes to be in the spin-up and spin-down states at later times behave like $-i \sin (\Omega t)$ and $\cos (\Omega t)$ respectively, where $\Omega$ 's expansion in powers of $\epsilon$ starts with $\epsilon E_{0}^{(1)} / 2$. The spin precesses in the tangent plane to the cylinder (i.e., perpendicularly to the magnetic field) while the particle oscillates from $-b$ to zero and back.

\section{Concluding Remarks}

We have studied the problem of the motion of a spin- $1 / 2$ particle on a cylinder, in the presence of a radial magnetic field. A non-standard perturbative analysis, applicable to any perturbation of the harmonic ascillator, led to a recursion relation for the wavefunction and energy corrections, Eqs. (26) and (27) respectively, with explicit results for the ground state in Eqs. (34) - (37) and Figures 3 , 4 It is worth emphasizing that the radial magnetic field of the problem can be approximated in the laboratory, as pointed out in the first footnote. 


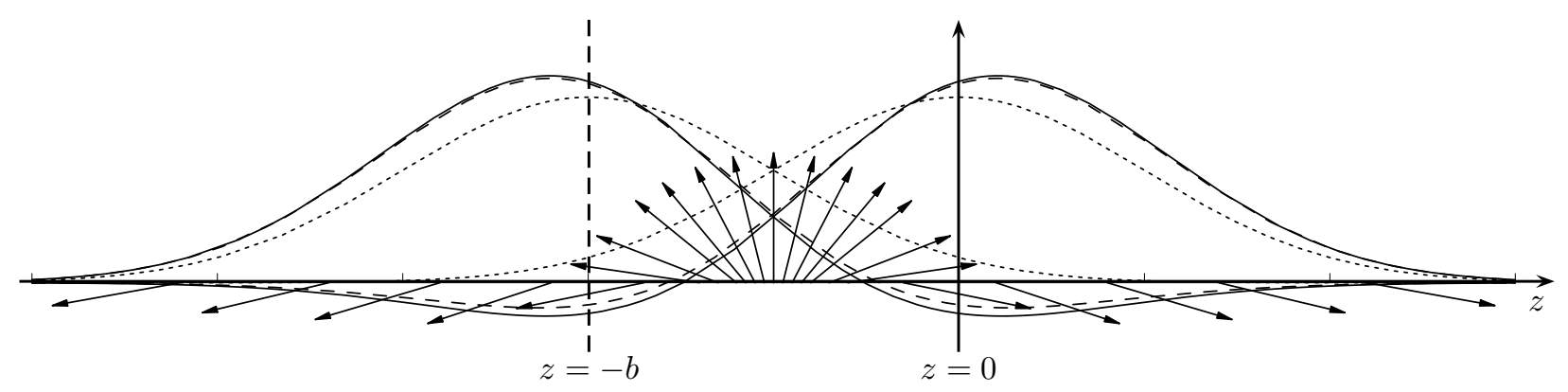

Figure 3: The spin components $Z_{\mathrm{s}+}, Z_{\mathrm{s}-}$ and the corresponding spin configuration. The dotted curves give the zeroth-order result, i.e., two gaussians, centered at $z=0$ and $z=-b=-2$. The dashed curves include corrections up to first order while the solid ones up to second $(\epsilon=0.5-$ a rather large value was used to make the effect visible). The integrals in Eqs. (36), (37) have been evaluated numerically. The spin configuration shown includes quadratic corrections. Due to the symmetry, the spin points always along $\hat{\rho}$ (upwards in the figure) at $z=-b / 2=-1$, while it tends to $\pm \hat{z}$ as $z$ tends to $\pm \infty$. The perturbation causes a zero in each component (for finite $z$ ) - the spin crosses the $z$ axis at those points. As a result, the two gaussians are pushed apart while the width of the central region, where the spin points up, is reduced.

We end with a comment on the form of the unperturbed hamiltonian used, Eq. (44). When dealing with the motion of a quantum particle on a surface, one can use a 3-D Laplacian in the hamiltonian and constrain the motion of the particle on the surface using a steep confining potential in the radial direction. It is well known that, in this approach, which seems to be the one appropriate for practical applications, there is an induced potential for the motion along the surface, proportional to the square of the difference between the two principal curvatures of the surface (see, e.g., 3, 14 and references therein). In our case, this is a constant which only shifts the energy eigenvalues. Nevertheless, an obvious extension of our problem here would be the study of the motion on the surface of a slightly curved cylinder, in which case the above mentioned induced potential would have to be taken into account.

\section{A First Order Corrections to the Ground State: the Standard Treatment}

We outline here the standard first order perturbative analysis of the problem, deriving the corrections to the ground state. Given that the zeroth order spectrum is degenerate, we need to first diagonalize the interaction hamiltonian in each degenerate subspace. One easily sees that the interaction only connects the pairs of eigenstates $|n, \ell,+\rangle,|n, \ell+1,-\rangle$. The appropriate zeroth order basis is given by the symmetric and antisymmetric linear combinations

$$
\begin{aligned}
\left|n_{\ell}^{\mathrm{s}}\right\rangle \equiv\left|n_{\ell}^{0}\right\rangle & =\frac{1}{\sqrt{2}}(|n, \ell,+\rangle+|n, \ell+1,-\rangle) \\
\left|n_{\ell}^{\mathrm{a}}\right\rangle \equiv\left|n_{\ell}^{1}\right\rangle & =\frac{1}{\sqrt{2}}(-|n, \ell,+\rangle+|n, \ell+1,-\rangle) .
\end{aligned}
$$

Notice that we use the label $\ell$ for states that are equally localized at $-\ell b$ and $-(\ell+1) b$. The reason for renaming the states with numerical superscripts, instead of letters, will become apparent below. The expectation value of $H_{\mathrm{int}}$ in these states reproduces our result (34) for the first order correction to the energy. It is interesting to see how the first order correction to the wavefunction, conventionally given by an infinite sum, is brought 


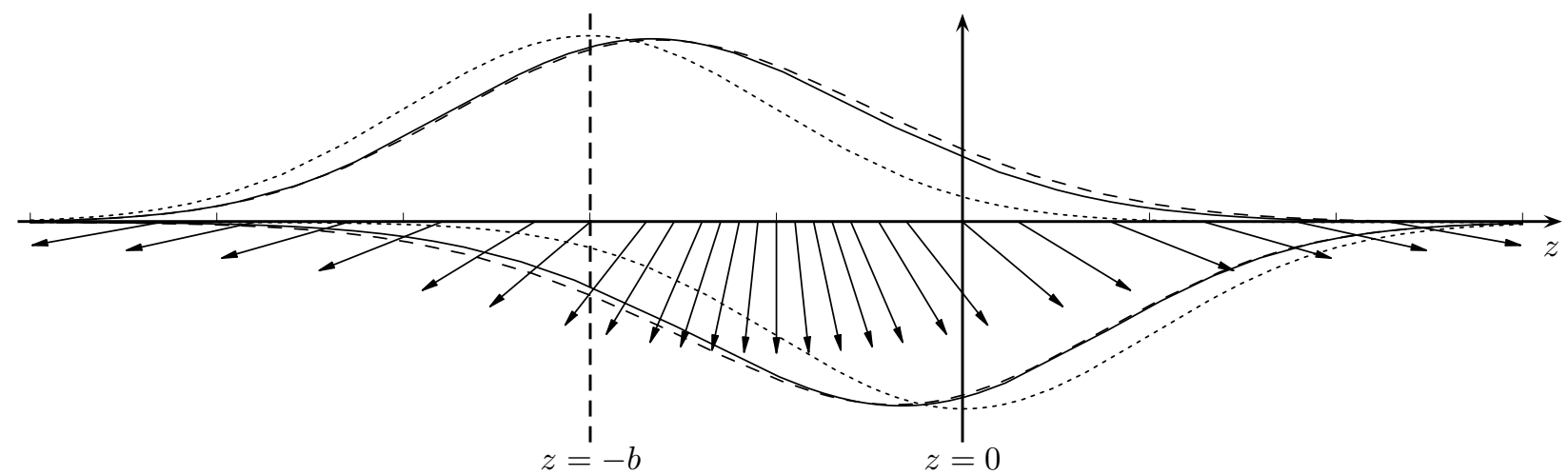

Figure 4: The spin components $Z_{\mathrm{a}_{+}}, Z_{\mathrm{a}_{-}}$and the corresponding spin configuration. Notation and parameter values are as in Fig. 3 Due to the antisymmetry, the spin points always along $-\hat{\rho}$ (downwards in the figure) at $z=-b / 2=-1$, while it tends to $\pm \hat{z}$ as $z$ tends to $\pm \infty$. The perturbation pushes the two gaussians together while the width of the region where the spin points downwards is increased.

into the closed form (36). The matrix elements of $H_{\mathrm{int}}$ in the above basis are

$$
\begin{aligned}
\left\langle n_{\ell}^{0}\left|H_{\mathrm{int}}\right| m_{\ell^{\prime}}^{0}\right\rangle & =\epsilon\left\langle n_{\ell} \mid m_{\ell+1}\right\rangle \delta_{\ell \ell^{\prime}} \delta_{\tilde{n} \tilde{m}} \\
\left\langle n_{\ell}^{0}\left|H_{\mathrm{int}}\right| m_{\ell^{\prime}}^{1}\right\rangle & =\epsilon\left\langle n_{\ell} \mid m_{\ell+1}\right\rangle \delta_{\ell \ell^{\prime}} \delta_{\tilde{n}, \tilde{m}+1} \\
\left\langle n_{\ell}^{1}\left|H_{\mathrm{int}}\right| m_{\ell^{\prime}}^{1}\right\rangle & =-\epsilon\left\langle n_{\ell} \mid m_{\ell+1}\right\rangle \delta_{\ell \ell^{\prime}} \delta_{\tilde{n} \tilde{m}},
\end{aligned}
$$

where $\left\langle n_{\ell} \mid m_{\ell+1}\right\rangle$ is the overlap between SHO eigenstates $|n\rangle,|m\rangle$, at a distance $b$ apart and $\tilde{n}$ is the parity of $n$. Specifying to the symmetric ground state and taking $\ell=0$, we find the first order correction

$$
\left|0_{0}^{0}\right\rangle^{(1)}=\sum_{k=1}^{\infty} \frac{\left\langle 0_{0} \mid k_{1}\right\rangle}{k}\left|k_{0}^{\tilde{k}}\right\rangle .
$$

We see that only states with $\ell=0$ contribute. Furthermore, when $k$ is even, only the symmetric state contributes while for $k$ odd, only the antisymmetric one does. Using the fact that

$$
\left\langle 0_{0} \mid k_{1}\right\rangle=\frac{1}{\sqrt{k !}}\left(-\frac{b}{\sqrt{2}}\right)^{k} e^{-\frac{b^{2}}{4}}
$$

we find for the spin-up component of the correction

$$
\left|0_{0}^{0},+\right\rangle^{(1)}=\sum_{k=1}^{\infty} \frac{(-1)^{k}}{k \sqrt{k !}}\left(\frac{b}{\sqrt{2}}\right)^{k} e^{-\frac{b^{2}}{4}}|k\rangle,
$$

which implies

$$
\begin{aligned}
b \frac{\partial}{\partial b}\left(e^{\frac{b^{2}}{4}}\left|0_{0}^{0},+\right\rangle^{(1)}\right) & =\sum_{k=1}^{\infty} \frac{(-1)^{k}}{\sqrt{k !}}\left(\frac{b}{\sqrt{2}}\right)^{k}|k\rangle \\
& =e^{\frac{b^{2}}{4}}\left(\left|0_{1}\right\rangle-\left|0_{0}\right\rangle\right) .
\end{aligned}
$$

Integrating back w.r.t. $b$ and changing integration variable we recover our earlier result (32) (similarly for the spin-down component).

\section{Acknowledgments}

The first author (C. C.) would like to acknowledge partial support from CONACyT projects 32307-E, 41208-F, and DGAPA-UNAM projects IN 119792, IN 114302. 


\section{References}

[1] P. P. Banderet. Helv. Phys. Acta, 19:503, 1946.

[2] M. De Crombrugghe and V. Rittenberg. Supersymmetric Quantum Mechanics. Ann. Phys., 151:99-126, 1983.

[3] R. C. T. da Costa. Quantum Mechanics of a Constrained Particle. Phys. Rev., 23:1982-1987, 1981.

[4] E. D'Hoker and L. Vinet. Supersymmetry of the Pauli Equation in the Presence of Magnetic Monopole. Phys. Lett., 137B:72-76, 1984.

[5] P. A. M. Dirac. Proc. Royal Soc., A133:60, 1931.

[6] M. Encinosa and R. H. O'Neal. Quantum Particle Constrained to a Curved Surface in the Presence of a Vector Potential. quant-ph/9908087.

[7] K. Ford and J. A. Wheeler. Application of Semiclassical Scattering Analysis. Ann. Phys., 7:287-322, 1959.

[8] A. S. Goldhaber. Role of Spin in the Monopole Problem. Phys. Rev., 140:B1407-1414, 1965.

[9] Harish-Chandra. Motion of an Electron in the Field of a Magnetic Pole. Phys. Rev., 74:883-887, 1948.

[10] R. W. Haymaker and A. R. P. Rau. Supersymmetry in Quantum Mechanics. Am. J. Phys., 54:928-936, 1985.

[11] R. Jackiw. Dynamical Symmetry of the Magnetic Monopole. An. Phys., 129:183-200, 1980.

[12] Y. Kazama, C. N. Yang, and A. S. Goldhaber. Scattering of a Dirac Particle with Charge Ze by a Fixed Magnetic Monopole. Phys. Rev. D, 15:2287-2299, 1977.

[13] L. D. Landau and E. M. Lifshitz. Quantum Mechanics. Pergamon Press, 1981. Third edition.

[14] P. C. Schuster and R. L. Jaffe. Quantum Mechanics on Manifolds Embedded in Euclidean Space. hep-th/0302216.

[15] J. Schwinger, K. A. Milton, W. Tsai, Jr. L. L. DeRaad, and D. C. Clark. Nonrelativistic Dyon-Dyon Scattering. Ann. Phys., 101:451-495, 1976.

[16] S. Takagi and T. Tanzawa. Quantum Mechanics of a Particle Confined to a Twisted Ring. Progr. Theor. Phys., 87:561-568, 1992. 